\title{
The Behavior of Spontaneous Volunteers: A Discrete Choice Experiment on the Decision to Help
}

\author{
Sebastian Lindner \\ Martin Luther University Halle-Wittenberg \\ sebastian.lindner@wiwi.uni-halle.de
}

\begin{abstract}
Modern communication technology has enabled new ways to exchange information and is one of the main drivers for citizens participation in disaster response. During the last decades, so-called spontaneous volunteers have become an important resource in coping with disasters. However, their unpredictable behavior has also led to several problems. Disaster managers urgently need insights into volunteers behavior to effectively use the offered potential. To gain and provide these insights into explaining what drives the decision to help, we performed a discrete choice experiment based on previously identified behavior-affecting attributes. Our results indicate that attributes like the scale of the disaster and the media coverage are among the most important factors in the decision to help. The model correctly predicts volunteers scenario-specific decisions with an accuracy of $65 \%$. Hence, the experiment offers valuable insights into volunteers behaviors for disaster research and is a sound foundation for decision support for disaster management.
\end{abstract}

\section{Introduction}

Modern communication technologies and, accordingly, the rising interconnection of society have enabled new ways of information retrieval and information dissemination. Technologies like social media have led to new opportunities for businesses and also the public sector. Since the advent of social media, this media has also affected the ways how people perceive disasters and how people interact and exchange information during disasters. These new opportunities have also led to a massive increase in citizens participation in disasters [1]. Consequently, social media has become a substantial topic in disaster research.

During the last decade, several disasters have shown increasing numbers of citizens helping spontaneously in mitigating disaster scales. These so-called spontaneous

\author{
Christoph Herrmann \\ Martin Luther University Halle-Wittenberg \\ christoph.herrmann@wiwi.uni-halle.de
}

volunteers, sometimes also referred to as emergent groups, are people who are not affiliated with a disaster response or humanitarian organization and are, thus, usually not trained in disaster response [2, 3]. In a disaster context, spontaneous volunteers assist official disaster relief forces by, e.g., filling sandbags or clearing up locations [4, 5].

According to disaster managers, scales of many disasters would have turned out to be much more dramatic without the help of spontaneous volunteers [3, 6]. However, these volunteers often coordinate their actions mainly based on information retrieved from social media platforms such as Facebook or Twitter. This information is often disseminated and collected by the citizens themselves and are, thus, subjective or even wrong. Self-coordination has led to several problems, e.g., overloading operating sites in main areas while peripheral areas are understaffed [7, 8]. Although people have been willing to help, they have been rejected at overloaded operating sites by officials. That has led to their frustration [2, 9] as well as to a potential decrease in their willingness to help in future disasters.

Not only have spontaneous volunteers been frustrated, but also official disaster management has been confronted by this massive participation and the inability to intercept this situation [4, 10]. The undeniable importance of spontaneous volunteers and the problems related to their self-coordination have triggered many researchers to develop concepts to integrate the coordination of spontaneous volunteers into official disaster management [2, 10, 11, 12]. Additionally, because not all people want to be or can be led or coordinated by official disaster management, disaster managers urgently need insights and information on how spontaneous volunteers act and react in particular situations to promptly intervene and plan with these resources instead of being overwhelmed.

To provide these insights, this paper aims to elicit the preferences of spontaneous volunteers methodically grounded on discrete choice experiments. The analysis leads to the identification of effects of 
behavior-influencing attributes on the willingness to help as well as to influences on scenario-specific decision-making. The resulting model can forecast a volunteer's decision within different scenarios.

Our results give an understanding of which factors influence the behavior, which is the main driver of spontaneous volunteering and how they affect individuals decisions to help. The model can forecast an individual's scenario-specific decision whether and where to help. Implementing this model and providing individual decisions within an agent-based simulation framework [13, 14] enables the observation of the emergent behavior of citizens in disasters. Hence, this can serve as a foundation to developing a decision support system for disaster management that, e.g., enables the monitoring of disaster-related key performance indicators, such as operating site utilization and allows developing strategies for spontaneous volunteers in the preparedness phase.

\section{Theoretical background and related work}

The behavior and especially the motivation of organized, or affiliated, volunteers have already intensively been investigated [15, 16, 17, 18, 19]. However, we focus on spontaneous instead of planned volunteers as their behavior is, at this point, unpredictable and they have caused problems in past disasters.

A major part of disaster-related research regarding citizens' participation in disasters constitutes the analysis of citizens behaviors and interactions within social networks and, thus, focus on so-called digital volunteers [1, 2, 3, 6, 7]. Whereas the efforts of spontaneous volunteers that physically help at operating sites and particularly the analysis of their behaviors are still relatively unexplored fields, researchers have discovered that spontaneous volunteers need proper coordination and an integration in official disaster management based on the previously described problems [8, 9, 4, 10]. Therefore, many researchers have come up with ideas and IT-supported coordination approaches, which constitute a major part of the research area. Furthermore, some researchers have already begun to analyze the drivers of spontaneous volunteering to provide insights into their behaviors and motivations [11, 20, 21, 22]. For instance, [14] have identified 25 attributes that affect the behavior of spontaneous volunteers. Although some researchers have begun to analyze drivers of spontaneous volunteering to provide insights, forecasting the behavior and actual actions of spontaneous volunteers in specific scenarios has, to the best of our knowledge, not yet been considered in disaster research. However, these study results serve as a sound foundation for this paper. Nonetheless, there are results from psychology and behavioral economics on the willingness to help in general. For instance, Amato discusses the influence of personality and social network involvement on the helping behavior on a general level [23]. Amato distinguishes between three types of helping activities: formal planned helping, informal planned helping, and spontaneous helping [23]. He concludes that the greatest number of helping activities is planned. Furthermore, he emphasizes that the willingness to participate in planned helping is more predictable than the willingness to help spontaneously. For this reason, the motivation of our approach is to improve the predictability of spontaneous volunteering.

Barraket et al. performed a comprehensive survey that gives first insights into motivational, intentional, and network-related aspects of spontaneous volunteering [11]. However, they do so only in a descriptive way.

Seo et al. strengthen the position that media can influence the willingness to help after a disaster [12]. Moreover, they emphasize the importance of media messages as a mediator to inform the public about the necessary actions needed to reduce harm. Besides, economists often investigate charity and donation behavior in the context of volunteering behavior. Donation activities are not spontaneous. Although the general research analyzes the effect of incentives on helping behavior like blood donation and investigates the so-called crowding-out effect [24, 25. 26], it only takes a shallow look at the actual triggers to donate. Considering the huge differences between the research on affiliated volunteers and that of spontaneous volunteers [22] the insights from planned helping cannot be adopted for spontaneous helping. Thus, insights into spontaneous helping require a comprehensive analysis.

Due to the nature of spontaneous volunteers in the context of natural disasters, one issue in investigating spontaneous volunteers behavior is that it is only observable if disasters occur. In such a situation, it is not practicable to observe or survey the spontaneous volunteers behavior and their decision to help. To overcome this issue, it is required to investigate the behavior and their decisions in an experimental frame with realistic simulated scenarios. Therefore, we propose a discrete choice experiment as an appropriate method to elicit the preferences of spontaneous volunteers decisions [27, 28, 29]. Within recent decades, the use of discrete choice experiments has increased in areas like health economics, transport economics, ecological economics, marketing efforts, and many 
more areas [29, 30, 31, 32]. However, there is no research using discrete choice experiments to identify the main drivers of potential spontaneous volunteers in the context of disaster response.

The lack of existing comprehensive investigations into spontaneous volunteers willingness to help and their decisions to physically help within different scenarios has motivated our effort to provide novel insights into spontaneous volunteer behaviors for research and practice. Thereby, the results of our discrete choice experiment can provide comprehensive data for, e.g., decision support systems for disaster management.

\section{Experimental design}

In conducting a discrete choice experiment, there are several steps to pass through to get valid results [33, 34].

Firstly, we have to decide how many alternatives will be given in the choice set [34, 35]. Since we are interested in insights into why people will or will not help in different scenarios, we also examine the decision not to help and provide three alternatives: two scenarios with different combinations of attribute levels and one opt-out alternative (see Figure 11). The participant has to choose one alternative: either one of two helping scenarios or the choice to not help at all. This allows the examination of the influence of different attributes on the decision and on the opportunity not to help. Veldwijk et al. find that including an opt-out can structurally change the results of the experiment since this reduces the number of missing data related to not being able to choose one of the alternatives [36]. Furthermore, Ryan and Skåtun figure out that a correctly included opt-out alternative is very important to illustrate a real-life situation [37].

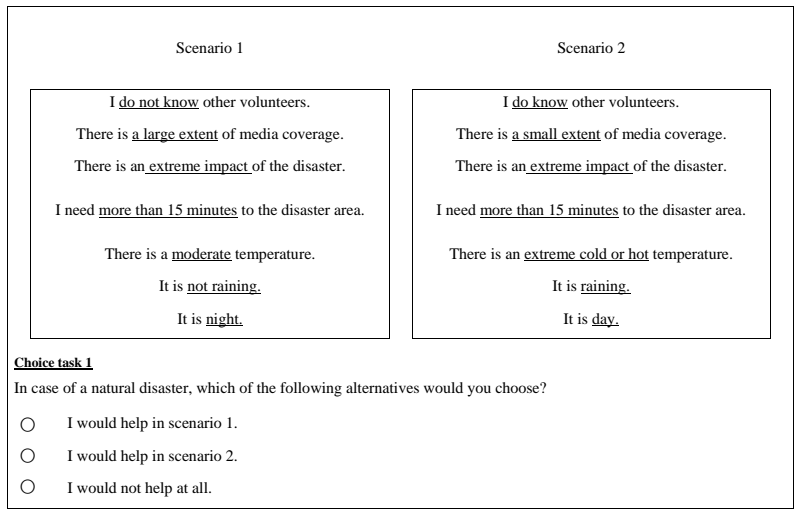

Figure 1. Example of a choice task

Secondly, we have to define the number of and the attributes themselves. There is an trade-off between including as many attributes as possible and the feasible cognitive load of the participants. The number of attributes, which should be incorporated into this step, varies in the literature. Maddala et al. propose to include only the key attributes in a decision task [38]. Louviere et al. find that the influence of the number of attributes on completion time is small [39]. Moreover, typically the number of attributes vary in the range from 4 to 8 [40], whereas more than 6 attributes can decrease the completion rate [39]. There are several ways to identify attributes affecting spontaneous volunteers willingness to help. One way is the identification of attributes based on a qualitative analysis of a literature review [41]. A literature review on factors that affect spontaneous volunteers behaviors has already found 25 potential attributes and serves as the foundation for our study.

To reduce the number of attributes given in the study, we have firstly excluded attributes that cannot be measured (e.g. attribute: randomness) or that do not directly affect the behavior (e.g. attributes: resources and traveling). To further reduce the number of attributes, we have secondly surveyed a group of experts (disaster researchers, people who have spontaneously helped in disasters before) to identify the main influences on the decision to help or not help. The overall process has led to 7 attributes that have been chosen for our further investigation.

The attributes and their associated levels are shown in Table 1 the first written level is the reference level.

Table 1. Selected Attributes

\begin{tabular}{|c|c|c|}
\hline attribute & levels & description \\
\hline impact & small/big & $\begin{array}{l}\text { How is the scale of the } \\
\text { disaster? }\end{array}$ \\
\hline friends & no/yes & Do friends already help? \\
\hline exp.time & short/long & $\begin{array}{l}\text { What is the expenditure } \\
\text { of time to get to the } \\
\text { operating site? }\end{array}$ \\
\hline daytime & night/day & $\begin{array}{l}\text { The daytime of the } \\
\text { present scenario. }\end{array}$ \\
\hline temp & extreme/normal & $\begin{array}{l}\text { The temperature of the } \\
\text { present scenario. }\end{array}$ \\
\hline media & low/high & $\begin{array}{l}\text { How is the media } \\
\text { coverage? }\end{array}$ \\
\hline rain & no/yes & Is it raining? \\
\hline
\end{tabular}

Thirdly, to limit the number of choice sets appropriately, we decided to give each attribute two levels [42]. We did so for two reasons: The levels have to be as relevant and as easy to understand as possible and, the number of levels should be equal for all attributes because individuals weigh attributes with more levels higher [33, 43]. Due to the local conditions of the respondent's city, the levels of expenditure of time have been translated to more resp. less than 15 minutes. 
Therefore, we indicate, as represented in Table 1 the level of the attribute impact as small and big. Seo et al. argue that the scale of a disaster is the main influence on the willingness to help spontaneously [12]. We also include personal connections [11, 21] as an attribute in the analysis because several psychological studies emphasize the role of personal connections in the context of helping. Additionally, the expenditure of time to get to operating sites [44, 21] is a relevant attribute. Although the influence of weather conditions and the time of day is discussed contradictorily in the literature, we include attributes like temperature, precipitation and whether the volunteers would be working during the day or night when help is needed to test if an influence exists [14]. Seo et al. address the role of media coverage [12]. For this reason, we also examine the effect of information by media coverage. Moreover, we decided to include individual-specific variables in our analysis. We incorporate the gender of our participants into the survey to test if there are any gender-specific differences. Moreover, we partly deduce the willingness to help by adding the willingness to install a coordination app as well as previous experiences in helping and the willingness to spend time to help on the participants days off.

Lastly, after setting the survey frame, we had to identify the number and composition of the choice sets to estimate the main effects of specific attributes. Therefore, we use the R-package support.CEs [45]. The $L^{M A}$ method is used to create the experimental design directly from a symmetric orthogonal main-effect array with $M$ times $A$ columns of $L$ level factors [46]. Hence, we have an orthogonal main-effect array with 2 times 7 columns of 2 levels to create a choice set with 2 alternatives of 7 attributes with 2 levels. We do not have to include the opt-out alternative the levels of the attributes do not vary over the choice sets and, thus, we only have two alternatives in the main-effect array. The support.CEs' function divides the choice set into subsets of the choice sets [45]. Our experimental design contains 32 different choice tasks which are divided into five questionnaires. Figure 1 displays an example of a choice task. The participants had an introduction into the topic where we have explained the given situation and the scenario. We have focused on a flood scenario to eliminate disaster type effects and, also, because floods are among the most realistic disasters at the location of the survey. Furthermore, we have also explained scenario-related typical tasks like filling sandbags, distribute food and drinks and clean up operating sites. We present two alternatives with their corresponding attributes and levels in boxes and underline the key characterizations. Underneath the boxes, the participants have to select which of the three alternatives they choose (single-choice).

After developing the experimental design we collected the following data: we conducted two samples, one in January 2018 (Sample 1) and another one in October 2018 (Sample 2). In Sample 1, 170 undergraduate students in an introductory statistics course took part. In general, we had 1360 ternary decisions which make 4080 observations. Since we only had a small number of responses where no or more than one alternative was chosen, we decided to discard these responses [47]. After reviewing the data, we ended up with 3492 observations. Additionally, 311 undergraduate students in another introductory statistics course took part in Sample 2. Hence, we have 2488 ternary choices that result in 7464 observations.

Subsequent to collecting the surveys and sorting out those with missing data, Sample 1 was used to estimate the utility function defined in Equation 1 It is used to evaluate the accuracy of our model that will be presented in Section 4. To analyze our data, we have to derive a utility function which captures all possible attributes and individual aspects that could influence the decision

\section{Model framework}

Since we are using a discrete choice experiment to retrieve the attributes that affect spontaneous volunteers decision to help, we consider that each individual maximizes her/his utility if she/he faces a decision [48, [49, 28]. These assumptions follow the random utility framework of McFadden [28]. Hence, each individual chooses the alternative with the highest personal benefit. We define the following utility function. The utility of an individual $i$ in alternative $j$ and choice task $t$ is:

$$
U_{i j t}=\alpha_{i}^{\prime} z_{j t}+\beta_{j}^{\prime} x_{i}+\varepsilon_{i j t},
$$

where $z_{j t}$ describes a vector of attributes (alternative-specific variables) and $\alpha_{i}^{\prime}$ are the corresponding vectors of coefficients. Since we have repeated measurements for each individual, we can take the heterogeneity between individuals into account. Therefore, we use a step-wise reduction of random parameters based on likelihood ratio tests. We end up with 3 random parameters (impact, friends, daytime).

The other alternative-specific variables are estimated at the mean. By $x_{i}$ we define the individual-specific control variables. Here, $\beta_{j}$ are the associated coefficients which are estimated for each alternative. Finally, $\varepsilon_{i j t}$ is the independently identically extreme-value-type-1 distributed error term. We imply 
Table 2. Model evaluation method

\begin{tabular}{lllll|l}
\hline & \multicolumn{5}{c}{ predicted outcomes } \\
& & $\mathrm{S} 1$ & $\mathrm{~S} 2$ & $\mathrm{O}$ & $\sum$ \\
\hline \multirow{2}{*}{ observed } & $\mathrm{S} 1$ & $T P_{1}$ & $F P_{21}$ & $F N_{1}$ & $N_{r 1}$ \\
outcomes & $\mathrm{S} 2$ & $F P_{12}$ & $T P_{2}$ & $F N_{2}$ & $N_{r 2}$ \\
& $\mathrm{O}$ & $F P_{1}$ & $F P_{2}$ & $T N$ & $N_{r 3}$ \\
\hline & $\sum$ & $N_{c 1}$ & $N_{c 2}$ & $N_{c 3}$ & $N$ \\
\hline
\end{tabular}

that an individual chooses the alternative with the highest utility in a decision task.

After estimating $\alpha_{i}^{\prime}$ and $\beta_{j}^{\prime}$, we validate our model by calculating utilities for all alternatives and individuals. We take the alternative with the highest estimated utility as the individuals estimated choice. Additionally, each individual faces three alternatives. This result is a multinomial outcome variable. To evaluate the prediction accuracy of our model, we compare the observed outcomes with the predicted outcomes. Cohen uses a similar procedure to measure the agreement for nominal scales [50].

Table 2 shows the possible cases which occur by comparing the observed and predicted outcomes. We particularly distinguish between four general results. $T P_{1}, T P_{2}$ we consider a true positive situation where the observed and predicted outcomes match each other and the prediction is a positive outcome. In our case, it means that the participant is predicted to help in a specific situation. In comparison, $T N$ is a true negative situation. The only difference to a true positive situation is that the outcome is predicted as a negative one so that $F P$ and $F N$ (false positive and false negative) define a result where the observed and predicted outcomes do not match each other. Furthermore, we derive four measures to evaluate our model. These measures are recommended by McFadden [51]. First, we can calculate the overall predictive precision (accuracy) which we consider as:

$$
a c c=\frac{T P_{1}+T P_{2}+T N}{N} .
$$

the accuracy that is defined as the matches between observed and predicted outcomes divided by the number of observations. Hence, we can accurately interpret the probability of a true prediction. Second, we can measure the precision of our model:

$$
\text { pre }=\frac{T P_{1}+T P_{2}}{N_{c 1}+N_{c 2}} .
$$

by constraining on the positive predictions. Precision is the conditional probability that thethe levels of the attributes outcome of an individual is predicted as a positive outcome and, thus, is correctly predicted. Third, we can examine the sensitivity of our model:

$$
\text { sen }=\frac{T P_{1}+T P_{2}}{N_{r 1}+N_{r 2}} .
$$

In contrast to precision, sensitivity denotes the conditional probability that an observed outcome of an individual is correctly predicted as a positive outcome. And fourthly, we can compute the specificity of our model:

$$
\text { spe }=\frac{T N}{N_{r 3}} .
$$

Specificity is the opposite of sensitivity. Therefore, specificity is defined as the conditional probability that an observed outcome is correctly predicted as a negative one. These measures allow us to identify the strengths and weaknesses of our model. In the next step, we will take a closer look at the results of our study.

\section{Results}

Table 3 presents the estimation results of the attributes and random parameters. We consider the random parameters as normally distributed. However, we select only three random parameters (impact, friends, daytime), based on the results of several likelihood ratio tests. We set up a 5\% significance level. All parameters are significant because the $95 \%$ confidence interval does not include a zero. The impact of the disaster has the largest effect, followed by friends.

Seo et al. emphasize the effect of the disasters impact [12]. The effect of friends means that if friends are helping, the probability of the individuals helping increases. Amato finds a similar result [23]. He exposed that the social network of a person influences the spontaneous volunteering behavior. Furthermore, people prefer to help during the day. Hence, the probability of helping in a specific situation decreases if the alternative scenario is defined as helping at night. Additionally, normal temperatures have a positive 
Table 3. Estimation results of the attributes

\begin{tabular}{|c|c|c|c|}
\hline \multicolumn{4}{|c|}{ Alternative-specific variables: } \\
\hline impact & $0.8237 *$ & 0.0807 & $0.6654-0.9819$ \\
\hline friends & $0.6853^{*}$ & 0.0788 & $0.5308-0.8399$ \\
\hline exp.time & $-0.6321 *$ & 0.0782 & $-0.7853--0.4789$ \\
\hline daytime & $0.5814 *$ & 0.0772 & $0.4302-0.7327$ \\
\hline temp & $0.3001 *$ & 0.0706 & $0.1618-0.4385$ \\
\hline media & $0.2956^{*}$ & 0.0689 & $0.1606-0.4306$ \\
\hline rain & $-0.2370^{*}$ & 0.0726 & $-0.3793--0.0948$ \\
\hline \multicolumn{4}{|c|}{ Standard deviations of random parameters: } \\
\hline sd.impact & $1.0421 *$ & 0.1245 & $0.7980-1.2862$ \\
\hline sd.friends & $0.8621 *$ & 0.1219 & $0.6233-1.1010$ \\
\hline sd.daytime & $0.6583^{*}$ & 0.1198 & $0.4236-0.8931$ \\
\hline$N$ & 3492 & & \\
\hline McFadden $R^{2}$ & 0.2817 & & \\
\hline
\end{tabular}

impact on the decision to help. Schneider et al. chose a different approach to identify the temperatures effect on spontaneous helping. The people were in a specific environment where the temperature varies directly in four different treatments. Schneider et al. observed an unclear effect of temperature in this setting [52]. Furthermore, the extent of the media coverage has a positive effect. This finding is in line with Seo et al. who observe that media can increase the willingness to help [12]. In contrast, only two variables have a negative impact on the decision to help. The expenditure of time to get to the disaster area on the one hand and, the precipitation conditions on the other hand. This means that if the duration to get to the operating site is high, the probability to help decreases. We observe an equivalent effect for a raining situation. The random parameters indicate that the coefficients of impact, friends and daytime vary in population. These insights display that the other parameters (not random) do not vary in the population so that the effect is the same for each individual and, therefore, the mean.

The random parameters means and standard deviations are used to draw a distribution curve for each random parameter. Figure 2 shows the distributions of the random parameters. The distributions of the impact and friends coefficients indicate that the probability to have a negative effect is $21 \%$. We obtain this probability by computing the area under the distribution curve for impact smaller than zero. One explanation for the impact variable is that a larger disaster results in higher probabilities of people willing to confront danger, which could be tested by including an attribute which illustrates the degree of willingness to put oneself in danger. The interpretation of the friends variable is the indication that spontaneous volunteers may not be aware that there are enough people at a certain
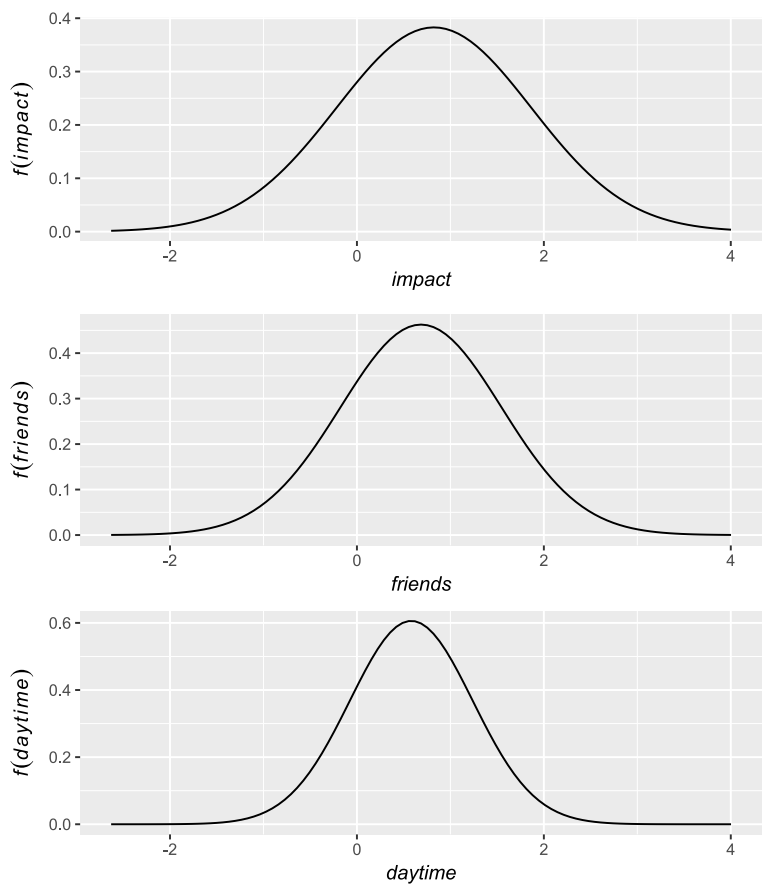

Figure 2. Distributions of the random parameters

operating site. To overcome this issue, we should add an attribute that indicates information about operating site utilization. However, the distribution of the daytime coefficient shows that the probability to have a smaller coefficient than zero is $19 \%$. Some individuals seem to prefer helping at night.

Amato found that planned helping behavior is generally driven by attitudinal, personality, and demographic variables [53]. This motivates us to take a closer look at the individual-specific effects displayed in Table 4. The coefficients are estimated for each 
Table 4. Estimation results of the individual-specific variables

\begin{tabular}{lccc}
\hline \multicolumn{4}{l}{ Individual-specific variables: } \\
& $\mathrm{S} 1$ & $\mathrm{~S} 2$ & $\mathrm{O}$ \\
\hline intercept & 0 & -0.2804 & $1.1402^{*}$ \\
gender & 0 & -0.0635 & -0.0159 \\
app - maybe & 0 & 0.4940 & $-0.7305^{*}$ \\
app-yes & 0 & 0.1966 & $-1.5858^{*}$ \\
helped & 0 & $-0.4227^{*}$ & $-1.0301^{*}$ \\
leisure.time & 0 & 0.0397 & $-0.1696^{*}$ \\
\hline
\end{tabular}

* coefficients significant at the $5 \%$ level

alternative where Scenario 1 (S1) serves as the reference level so that all individual-specific coefficients have to be zero. As Scenario 1 (S1) and Scenario 2 (S2) differ only in the attribute levels, there should be no significant differences between S1 and S2. In contrast, there should be significantly different effects for the opt-out $(\mathrm{O})$ alternative because the decision to choose between the reference level $\mathrm{S} 1$ and $\mathrm{O}$ depends to a large extent on the individual. We include five individual-specific variables plus intercept in the model. First of all, we control the effect of gender on the decision to help. The results show that there is no difference for male and female. This is in line with the literature [53, 54, 52]. Secondly, we look at the willingness to install a spontaneous volunteer coordination app. This partly captures the general willingness to help. We observe that the decision not to help decreases for participants who would maybe or certainly install such an app. We find a significant effect at the 5\% level for maybe installing the app ,and for installing the app. For S2, both coefficients are not significant. Additionally, we include the experience of the participants with previously helping in a disaster situation and label it helped. We observe a significant negative effect for the second alternative. Moreover, the variable helped has a negative effect on the decision not to help. This effect also is significant and means the probability to choose the opt-out alternative decreases if an individual has already helped in a disaster. Lastly, we observe a negative effect on choosing the opt-out alternative for people who report the willingness to help longer on their days off. This effect differs significantly from zero. We do not find a significant positive effect for Scenario 2. The four individual-specific variables app - maybe, app - yes, helped and leisure.time are all related to motivation and empathy. There are many contributions that confirm our findings that empathy and intrinsic motivation play a major role for explaining helping behavior [54, 55, 24].

To evaluate the goodness of fit of the model we can use the McFadden Pseudo- $R^{2}$ and the methods explained in Section 4 In Table 3, the McFadden
Pseudo- $R^{2}$ of the model is reported as approximately 0.28. McFadden determines a Pseudo- $R^{2}$ between 0.2 and 0.4 as a good fit [51]. The measure can be interpreted as the ratio of information gain when dividing the $\log$ likelihood of the full model by the log likelihood of the null model [51]. Because we are also interested in the predictive accuracy of our model, we compute the overall predictive accuracy, precision, sensitivity, and specificity of our utility function.

Table 5. Prediction results of both samples in percentages

\begin{tabular}{lllll}
\hline Sample1 & & \multicolumn{3}{c}{ Predicted outcomes } \\
& & S1 & S2 & O \\
\hline \multirow{2}{*}{ Observed } & S1 & $30.76 \%$ & $10.05 \%$ & $1.37 \%$ \\
outcomes & S2 & $9.62 \%$ & $31.53 \%$ & $1.98 \%$ \\
& O & $6.01 \%$ & $5.58 \%$ & $3.09 \%$ \\
\hline Sample 2 & & \multicolumn{3}{c}{ Predicted outcomes } \\
& & S1 & S2 & O \\
\hline \multirow{2}{*}{ Observed } & S1 & $31.19 \%$ & $9.59 \%$ & $3.49 \%$ \\
outcomes & S2 & $12.59 \%$ & $25.08 \%$ & $2.95 \%$ \\
& O & $6.78 \%$ & $5.38 \%$ & $2.95 \%$ \\
\hline
\end{tabular}

Table 5 shows the prediction results of Sample 1 which we use to estimate our model and Sample 2 which we use to validate our model. We illustrate the results in percentages by multiplying the results with 100 . The frequency for Sample 1 of the $T P_{1}$ cases is $30.76 \%$ and of the $T P_{2}$ cases is $31.53 \%$. In comparison, the frequency for Sample 2 of the $T P_{1}$ cases is $31.19 \%$ and of the $T P_{2}$ cases is $25.08 \%$. Hence, there are only small differences between both samples in frequencies of $T P_{1}$ and $T P_{2}$. Moreover, we predict for Sample 2 Scenario 1 a bit better than for Sample 1, whereas we have a loss in prediction for Scenario 2. Only approximately $3 \%$ of all cases are $\mathrm{TN}$ for both samples. In contrast, we observe that the opt-out alternative was chosen approximately $15 \%$ out of all cases. Furthermore, if we compare the frequencies of the $F P$ and $F N$ cases for both samples, we find nearly small differences in the range 0.14 to 2.97 percentage points.

Table 6. Evaluation measures in percentages

\begin{tabular}{llll}
\hline & Sample 1 & Sample 2 & $\Delta$ \\
\hline acc $\cdot 100 \%$ & $65.38 \%$ & $59.23 \%$ & $6.15 \mathrm{pp}$ \\
pre $\cdot 100 \%$ & $66.57 \%$ & $62.11 \%$ & $4.46 \mathrm{pp}$ \\
sen $\cdot 100 \%$ & $73.01 \%$ & $66.29 \%$ & $6.72 \mathrm{pp}$ \\
spc. $100 \%$ & $21.05 \%$ & $19.55 \%$ & $1.50 \mathrm{pp}$ \\
\hline
\end{tabular}

Finally, we calculate the measures to evaluate our model. Table 6 displays the evaluation measures. 
We also compute the percentages of the measures by multiplying them with 100 . First of all, we see that the overall predictive accuracy for Sample 1 is $65.38 \%$. Therefore, we can improve our prediction accuracy by assuming the utility function of Equation 11. Out of a statistical point of view, where the aim usually is to increase the prediction, the result indicates that the model works. Moreover, we only have a loss of 6.15 percentage points when we predict the outcomes of Sample 2 on the basis of the estimation results from Sample 1. The loss of precision between Samples 1 and 2 is 4.46 percentage points. For this reason, the conditional probability that a predicted positive outcome of an individual is correctly predicted as helping in the right scenario is nearly the same.

The sensitivity is between $66.29 \%$ for Sample 2 and $73.01 \%$ for Sample 1. Thus, both samples have a high value of sensitivity. These findings are very helpful if the interest is to predict an observed outcome correctly compared to the observed helping scenario. Additionally, this means that if we want to predict to which operating site an individual will go, we will have a high success rate. This can be helpful in coordinating spontaneous volunteers. However, another result is that the alternative-specific variables explain especially the scenarios' choice whereas the the individual-specific variables explain the choice of the out-put option. Lastly, our model performs very poorly in predicting the opt-out alternative. One reason could be that we cannot control for all individual-specific attributes which we have found in the literature. To increase the specificity of our model, more individual-specific variables need to be included. Selecting the opt-out alternative depends mostly on individual-specific variables shown in Table 4 and selecting scenario 1 or 2 depend on the alternative-specific variables.

\section{Conclusion}

Recent disasters have revealed the undeniable importance of spontaneous volunteers for supporting the mitigation of disaster scales. However, several problems regarding the help of spontaneous volunteers have led to the assumption that official disaster managers require insights into spontaneous volunteer behaviors to utilize these volunteers as a valuable resource and to avoid problems related to their support.

To gain these insights, we have developed and performed a discrete choice experiment on attributes that have been identified in a prior study. We have retrieved a model that can, within the frame of the experiments, predict the decisions to help in different scenarios with an accuracy of $65 \%$. The impact of individual attributes could be analyzed and compared to previous studies that have only partly focused on the attributes. Hence, the results can extend previous study results and give interesting insights into volunteers behaviors for practice and disaster research. Furthermore, the retrieved model is necessary to develop a simulation framework to forecast spontaneous volunteers' behaviors since it enables the individuals decision to help within a given scenario and, thus, allows observing emergent behaviors.

However, there are some limitations regarding the proposed experiment and model. Even though students (along with employees) have been the major group of spontaneous volunteers in recent disasters [11], we have exclusively surveyed the attitudes of students, which, thus, is a limiting factor regarding our outcomes. Furthermore, 25 behavior-affecting attributes had been identified within a literature review, but, due to the limitations of discrete choice experiments, we had to reduce the number of observed attributes to 7 , which we have done by questioning only some experts. To sum up, discrete choice experiments deliver accurate results for a small number of attributes and can, if extended to a more generic group of participants, deliver even more valuable insights into spontaneous volunteers behavior.

Nevertheless, an analysis of 7 alternative-specific plus 5 individual-specific variables has led to a large improvement in prediction accuracy. Moreover, according to Reunanen, the increase in accuracy by adding more variables in the model would probably be small [56]. Furthermore, the selection of many behavior-affecting attributes can lead to over-fitting the model [56]. Certainly, there are opportunities to improve the model. In order to achieve this goal, however, one would have to either a) detect the subset of the most behavior impacting attributes (e.g. step-wise regression [57]) and perform another discrete choice experiment with this subset to get a frugal model preferable for the sake of statistics, or b) one would have to make use of another statistical method that can take all attributes into consideration, or c) one would need to use a combination of both. Also important, the literature review has proposed deeper investigations on some of the attributes because it revealed contradictory statements to some of the attributes, and, thus, the effect of some attributes is still not clear. A structural equation model (SEM) seems to be promising since SEM enables a more flexible model, which also measures relationships between variables and takes mediator effects into account. SEM can, moreover, incorporate latent variables, which are usually mediators like motivation and empathy.

The analysis provides deep insights into the 
impacts of attributes on the willingness of spontaneous volunteers to help in disaster situations. These insights are helpful for practitioners. Furthermore, the proposed method and the study results are good foundations for other researchers to extend the investigation or to, e.g., implement the results in a decision support system for disaster managers. For instance, researchers could also emphasize the role of image concerns as a factor of motivation for an interesting next approach. We will address the proposed desiderata within our future research.

\section{References}

[1] A. L. Hughes and A. H. Tapia, "Social media in crisis: When professional responders meet digital volunteers," Journal of Homeland Security and Emergency Management, vol. 12, no. 3, pp. 679-706, 2015.

[2] M.-A. Kaufhold and C. Reuter, "The self-organization of digital volunteers across social media: The case of the 2013 european floods in germany," Journal of Homeland Security and Emergency Management, vol. 13, no. 1, pp. 137-166, 2016.

[3] C. Reuter, T. Ludwig, M.-A. Kaufhold, and V. Pipek, "Xhelp: Design of a cross-platform social-media application to support volunteer moderators in disasters," in Proceedings of the 33rd annual ACM conference on human factors in computing systems, pp. 4093-4102, ACM, 2015.

[4] T. Ludwig, C. Kotthaus, C. Reuter, S. van Dongen, and V. Pipek, "Situated crowdsourcing during disasters: Managing the tasks of spontaneous volunteers through public displays," International Journal of Human-Computer Studies, vol. 102, pp. 103-121, 2017.

[5] J. Whittaker, B. McLennan, and J. Handmer, "A review of informal volunteerism in emergencies and disasters: Definition, opportunities and challenges," International Journal of Disaster Risk Reduction, vol. 13, pp. 358-368, sep 2015.

[6] R. dos Santos Rocha, A. Widera, R. P. van den Berg, J. P. de Albuquerque, and B. Helingrath, "Improving the involvement of digital volunteers in disaster management," in International Conference on Information Technology in Disaster Risk Reduction, pp. 214-224, Springer, 2016.

[7] W. T. Coombs, "Digital naturals and the effects of social media on disaster communication," in State, society and national security: Challenges and opportunities in the 21st century, pp. 183-191, World Scientific, 2016.

[8] B. McLennan, J. Molloy, J. Whittaker, and J. Handmer, "Centralised coordination of spontaneous emergency volunteers: the ev crew model," The Australian Journal of Emergency Management, vol. 31, no. 1, pp. 24-30, 2016.

[9] U. Meissen, F. Fuchs-Kittowski, M. Jendreck, S. Pfennigschmidt, M. Hardt, and A. Voisard, "A general system architecture and design for the coordination of volunteers for agile disaster response.," in ISCRAM, 2017.

[10] G. Rauchecker and G. Schryen, "Decision support for the optimal coordination of spontaneous volunteers in disaster relief," in 15th International Conference on Information Systems for Crisis Response and Management, Mai 2018.

[11] J. Barraket, R. Keast, C. J. Newton, K. Walters, and E. James, "Spontaneous volunteering during natural disasters," tech. rep., The Australian Centre for Philanthropy and Nonprofit Studies, 2013. Working Paper No. ACPNS 61.

[12] M. Seo, S. Sun, A. J. Merolla, and S. Zhang, "Willingness to help following the sichuan earthquake," Communication Research, vol. 39, pp. 3-25, jan 2011.

[13] S. Lindner, S. Sackmann, and H. Betke, "Simulating spontaneous volunteers: A system entity structure for defining disaster scenarios," Proceedings of the Information Systems for Crisis Response and Management (ISCRAM), pp. 516-527, 2019.

[14] S. Lindner, H. Betke, and S. Sackmann, "Attributes for simulating spontaneous on-site volunteers," Proceedings of the 14th International Conference on Information Systems for Crisis Response and Management (ISCRAM), pp. 846-856.

[15] R. A. Cnaan and R. S. Goldberg-Glen, "Measuring motivation to volunteer in human services," The journal of applied behavioral science, vol. 27, no. 3, pp. 269-284, 1991.

[16] J. Bidee, T. Vantilborgh, R. Pepermans, G. Huybrechts, J. Willems, M. Jegers, and J. Hofmans, "Autonomous motivation stimulates volunteers work effort: A self-determination theory approach to volunteerism," Voluntas: International Journal of Voluntary and Nonprofit Organizations, vol. 24, no. 1, pp. 32-47, 2013.

[17] L. E. Miller, "Understanding the motivation of volunteers: An examination of personality differences and characteristics of volunteers' paid employment," Journal of Voluntary Action Research, vol. 14, no. 2-3, pp. 112-122, 1985.

[18] A. M. Omoto and M. Snyder, "Sustained helping without obligation: motivation, longevity of service, and perceived attitude change among aids volunteers.", Journal of personality and social psychology, vol. 68, no. 4, p. 671, 1995.

[19] J. C. Anderson and L. F. Moore, "The motivation to volunteer," Journal of Voluntary Action Research, vol. 7, no. 3-4, pp. 120-129, 1978.

[20] J. Twigg and I. Mosel, "Emergent groups and spontaneous volunteers in urban disaster response," Environment and Urbanization, vol. 29, no. 2, pp. 443-458, 2017.

[21] L. M. McDonald, M. Creber, H. Sun, and L. Sonn, "Developing public disaster communication for volunteer recruitment: understanding volunteer motivations," 2015.

[22] L. Kulik, L. Arnon, and A. Dolev, "Explaining satisfaction with volunteering in emergencies: Comparison between organized and spontaneous volunteers in operation protective edge," VOLUNTAS: International Journal of Voluntary and Nonprofit Organizations, vol. 27, pp. 1280-1303, Jun 2016.

[23] P. R. Amato, "Personality and social network involvement as predictors of helping behavior in everyday life," Social Psychology Quarterly, vol. 53, p. 31, mar 1990.

[24] N. Lacetera, M. Macis, and R. Slonim, "Rewarding volunteers: A field experiment," Management Science, vol. 60 , pp. 1107-1129, may 2014. 
[25] M. Touré-Tillery and A. Fishbach, "How to measure motivation: A guide for the experimental social psychologist," Social and Personality Psychology Compass, vol. 8, pp. 328-341, jul 2014.

[26] B. S. Frey and R. Jegen, "Motivation crowding theory," Journal of Economic Surveys, vol. 15, pp. 589-611, dec 2001.

[27] E. Lancsar, J. Louviere, and T. Flynn, "Several methods to investigate relative attribute impact in stated preference experiments," Social Science \& Medicine, vol. 64, pp. 1738-1753, apr 2007.

[28] D. McFadden, Conditional logit analysis of qualitative choice behavior, pp. 105-142. New York: Academic Press, 1973.

[29] K. E. Train, Discrete choice methods with simulation, vol. 1. Cambridge: Cambridge university press, 2009.

[30] E. W. de Bekker-Grob, M. Ryan, and K. Gerard, "Discrete choice experiments in health economics: a review of the literature," Health Economics, vol. 21, pp. 145-172, dec 2010 .

[31] D. Hoyos, "The state of the art of environmental valuation with discrete choice experiments," Ecological Economics, vol. 69, pp. 1595-1603, jun 2010.

[32] K. Zwerina, Discrete Choice Experiments in Marketing. Physica-Verlag HD, 1997.

[33] M. E. Kløjgaard, M. Bech, and R. Søgaard, "Designing a stated choice experiment: The value of a qualitative process," Journal of Choice Modelling, vol. 5, no. 2, pp. 1-18, 2012.

[34] E. Lancsar and J. Louviere, "Conducting discrete choice experiments to inform healthcare decision making," PharmacoEconomics, vol. 26, no. 8, pp. 661-677, 2008.

[35] J. DeShazo and G. Fermo, "Designing choice sets for stated preference methods: The effects of complexity on choice consistency," Journal of Environmental Economics and Management, vol. 44, pp. 123-143, jul 2002.

[36] J. Veldwijk, M. S. Lambooij, E. W. de Bekker-Grob, H. A. Smit, and G. A. de Wit, "The effect of including an opt-out option in discrete choice experiments," PLoS ONE, vol. 9, p. e111805, nov 2014.

[37] M. Ryan and D. Skåtun, "Modelling non-demanders in choice experiments," Health Economics, vol. 13, pp. 397-402, apr 2004.

[38] T. Maddala, K. A. Phillips, and F. R. Johnson, "An experiment on simplifying conjoint analysis designs for measuring preferences," Health Economics, vol. 12, no. 12, pp. 1035-1047, 2003.

[39] J. J. Louviere, R. T. Carson, L. Burgess, D. Street, and A. Marley, "Sequential preference questions factors influencing completion rates and response times using an online panel," Journal of Choice Modelling, vol. 8, pp. 19-31, sep 2013.

[40] R. T. Carson, J. J. Louviere, D. A. Anderson, P. Arabie, D. S. Bunch, D. A. Hensher, R. M. Johnson, W. F. Kuhfeld, D. Steinberg, J. Swait, H. Timmermans, and J. B. Wiley, "Experimental analysis of choice," Marketing Letters, vol. 5, pp. 351-367, oct 1994.

[41] T. M. Helter and C. E. H. Boehler, "Developing attributes for discrete choice experiments in health: a systematic literature review and case study of alcohol misuse interventions," Journal of Substance Use, vol. 21, pp. 662-668, mar 2016.
[42] J. J. Louviere, T. Islam, N. Wasi, D. Street, and L. Burgess, "Designing discrete choice experiments: Do optimal designs come at a price?," Journal of Consumer Research, vol. 35, pp. 360-375, aug 2008.

[43] J. Ratcliffe and L. Longworth, "Investigating the structural reliability of a discrete choice experiment within health technology assessment," International journal of technology assessment in health care, vol. 18, no. 1, pp. 139-144, 2002.

[44] F. Luqman and M. Griss, "Overseer: a mobile context-aware collaboration and task management system for disaster response," in 2010 Eighth International Conference on Creating, Connecting and Collaborating through Computing, pp. 76-82, IEEE, 2010.

[45] H. Aizaki, "Basic functions for supporting an implementation of choice experiments inR," Journal of Statistical Software, vol. 50, no. Code Snippet 2, 2012.

[46] F. R. Johnson, B. Kanninen, M. Bingham, and S. Özdemir, Experimental Design For Stated-Choice Studies, pp. 159-202. Dordrecht: Springer Netherlands, 2007.

[47] J. M. Brick and G. Kalton, "Handling missing data in survey research," Statistical methods in medical research, vol. 5, no. 3, pp. 215-238, 1996.

[48] F. J. Bahamonde-Birke, I. Navarro, and J. de Dios Ortúzar, "If you choose not to decide, you still have made a choice," Journal of Choice Modelling, vol. 22, pp. 13-23, mar 2017.

[49] J. J. Louviere, T. N. Flynn, and R. T. Carson, "Discrete choice experiments are not conjoint analysis," Journal of Choice Modelling, vol. 3, no. 3, pp. 57-72, 2010.

[50] J. Cohen, "A coefficient of agreement for nominal scales," Educational and Psychological Measurement, vol. 20, pp. 37-46, apr 1960.

[51] D. McFadden, Quantitative Methods for Analysing Travel Behaviour of Individuals: Some Recent Developments, ch. 13, pp. 279-318. Groom Helm, 1979.

[52] F. W. Schneider, W. A. Lesko, and W. A. Garrett, "Helping behavior in hot, comfortable, and cold temperatures," Environment and Behavior, vol. 12, pp. 231-240, jun 1980.

[53] P. R. Amato, "An investigation of planned helping behavior," Journal of Research in Personality, vol. 19, pp. 232-252, jun 1985 .

[54] P. A. V. Lange, M. Schippers, and D. Balliet, "Who volunteers in psychology experiments? an empirical review of prosocial motivation in volunteering," Personality and Individual Differences, vol. 51, pp. 279-284, aug 2011.

[55] M. H. Davis, K. V. Mitchell, J. A. Hall, J. Lothert, T. Snapp, and M. Meyer, "Empathy, expectations, and situational preferences: Personality influences on the decision to participate in volunteer helping behaviors," Journal of Personality, vol. 67, pp. 469-503, jun 1999.

[56] J. Reunanen, "Overfitting in making comparisons between variable selection methods," Journal of Machine Learning Research, vol. 3, no. Mar, pp. 1371-1382, 2003.

[57] P. Krishnaiah, "37 selection of variables under univariate regression models," Handbook of statistics, vol. 2, pp. 805-820, 1982. 